\title{
Delivery of Seasonal Malaria Chemoprevention With Enhanced Infection Prevention and Control Measures During the COVID-19 Pandemic in Nigeria, Burkina Faso and Chad: A Cross-sectional Study
}

\section{Charlotte Ward}

London School of Hygiene \& Tropical Medicine https://orcid.org/0000-0003-4223-7850

Abimbola Phillips

Malaria Consortium

\section{Olusola Oresanya}

Malaria Consortium

\section{Gloria Olisenekwu}

Oxford Policy Management

Ekundayo Arogunade

Oxford Policy Management

Azoukalné Moukénet

Université Cheikh Anta Diop de Dakar: Universite Cheikh Anta Diop de Dakar

Honoré Beakgoubé

Malaria Consortium

Vincent de Paul Allambademel

Universite de N'Djamena

Cheick Saïd Compaoré

Malaria Consortium

\section{Adama Traoré}

Malaria Consortium

Jean-Bosco Ouedraogo

Institut de Recherche en Sciences de la Sante

Yves Daniel Compaoré

Institut de Recherche en Sciences de la Sante

\section{Issaka Zongo}

Institut de Recherche en Sciences de la Sante

Laura Donovan ( $\sim$ l.donovan@malariaconsortium.org )

London, UK https://orcid.org/0000-0003-3676-2747 


\section{Monica Anna Decola \\ Malaria Consortium}

\section{Helen Smith}

Independent Consultant, International Health Consulting Services Ltd

\section{Kevin Baker}

Malaria Consortium

\section{Research}

Keywords: Seasonal malaria chemoprevention, malaria, COVID-19, infection prevention and control, children under five

Posted Date: August 30th, 2021

DOl: https://doi.org/10.21203/rs.3.rs-820778/v1

License: (c) (i) This work is licensed under a Creative Commons Attribution 4.0 International License. Read Full License

Version of Record: A version of this preprint was published at Malaria Journal on March 24th, 2022. See the published version at https://doi.org/10.1186/s12936-022-04091-z. 


\section{Abstract}

Introduction

Seasonal malaria chemoprevention (SMC) is a WHO-recommended intervention for children aged 3-59 months living in areas of high malaria transmission to provide protection against malaria during the rainy season. Operational guidelines were developed, based on WHO guidance, to support countries to mitigate the risk of coronavirus disease 2019 (COVID-19) transmission within communities and among community distributors when delivering SMC.

\section{Methods}

A cross-sectional study to determine adherence to infection prevention and control measures during two distribution cycles of SMC in Nigeria, Chad and Burkina Faso. Community distributors were observed receiving equipment and delivering SMC. Adherence across six domains was calculated as the proportion of indications in which the community distributor performed the correct action. Focus group discussions were conducted with community distributors to understand their perceptions of the infection prevention and control measures and barriers and facilitators to adherence.

Results

We observed community distributors in Nigeria $(n=259)$, Burkina Faso $(n=252)$ and Chad $(n=266)$ receiving infection prevention and control equipment and delivering SMC. Adherence to infection prevention and control indications varied. In all three countries, adherence to mask use was the highest (ranging from $73.3 \%$ in Nigeria to $86.9 \%$ in Burkina Faso). Adherence to hand hygiene for at least 30 seconds was low (ranging from $3.6 \%$ in Nigeria to $10.3 \%$ in Burkina Faso) but increased substantially when excluding the length of time spent hand washing (ranging from $36.7 \%$ in Nigeria to $61.4 \%$ in Burkina Faso). Adherence to safe distancing in the compound ranged from $5.4 \%$ in Chad to $16.4 \%$ in Nigeria. In Burkina Faso and Chad, where disinfection wipes widely available compliance with disinfection of blister packs for SMC was low (17.4\% in Burkina Faso and 16.9\% in Chad). Community distributors generally found the infection prevention and control measures acceptable, however there were barriers to optimal hand hygiene practices, cultural norms made social distancing difficult to adhere to and caregivers needed assistance to administer the first dose of SMC.

\section{Conclusion}

Adherence to infection prevention and control measures for SMC delivery during the COVID-19 pandemic varied across domains of infection prevention and control, but was largely insufficient, particularly for hand hygiene and safe distancing. Improvements in provision of equipment, early awareness raising and adaptations to make infection prevention and control measures more feasible to implement could increase adherence. 


\section{Introduction}

\section{Background/rationale}

The coronavirus disease 2019 (COVID-19) pandemic represents a huge threat to the maintenance of health service delivery globally. There is potential for direct mortality from COVID-19 and indirect mortality from preventable or treatable conditions such as malaria to increase dramatically. Previous epidemics have disrupted health systems and impacted on control programmes targeting specific diseases [1]. The World Health Organization (WHO) responded with operational guidance to support countries to reorganise and safely maintain access to high-quality, essential health services in the pandemic context [2], and specific guidance for tailoring malaria interventions in the COVID-19 response [3].

This includes seasonal malaria chemoprevention (SMC), a WHO-recommended intervention for children aged 3-59 months living in areas of high malaria transmission to provide protection during the rainy season [4]. SMC, the intermittent administration of sulfadoxine-pyrimethamine (SP) and amodiaquine (AQ) during the high transmission season, has been shown to be safe, feasible, effective and costeffective for the prevention of malaria among children under five $[5,6]$. The first daily dose of SPAQ is usually administered by a community distributor and the second and third daily doses of $A Q$ are left with the caregiver to administer. In 2020, Malaria Consortium's SMC programme targeted over 12 million eligible children in Nigeria, Burkina Faso and Chad across monthly cycles in the high transmission season [7].

Door-to-door delivery of SMC creates multiple opportunities for someone infected with severe acute respiratory syndrome coronavirus 2 (SARS-CoV-2), to transmit the virus through coughing, speaking, or exhaling, producing infective respiratory droplets that can be inhaled by those in close proximity. Infected droplets can also land on nearby surfaces or on SMC commodities such as SPAQ blister packs.

In response, operational guidance to support countries mitigate the risk of COVID-19 transmission when delivering SMC were developed [8]. Malaria Consortium also developed a job aid [9] and a training flipbook to explain the adaptations to the campaign and guide delivery of SMC during the pandemic. The guidance also specified that caregivers should administer all SPAQ doses, with the first doses of SP and $A Q$ administered under supervision of community distributors. Similar large-scale distribution campaigns have been implemented during previous epidemics $[10,11]$ but to our knowledge, no assessment to determine adherence to or perceptions of infection prevention and control measures during a pandemic has been done.

Based on Donabedian's model for assessing quality of care, we explored availability of infection prevention and control equipment (structure), adherence to infection prevention and control measures (process) and community distributor satisfaction with SMC delivery during COVID-19, including perceived quality and safety of the campaign (outcome) [12]. 


\section{Methods}

\section{Study design}

A cross-sectional study design was used to determine adherence to infection prevention and control measures during two cycles of SMC in September and October 2020 in Nigeria, Burkina Faso and Chad. Focus group discussions were conducted with community distributors to explore their perceptions of the infection prevention and control measures and barriers and facilitators to adherence.

Here, we report data from each country and present case studies to enable policy makers and SMC programme managers to observe the key challenges and successes relating to delivery of SMC during COVID-19 in areas where the SMC campaign has been implemented for at least one year.

\section{Study setting}

The study was conducted in urban centres and rural areas in Nigeria, Burkina Faso and Chad (Figure 1).

In Nigeria, the study was conducted in Sokoto state in the urban LGA Sokoto South and rural LGAs Tangaza and Silame. SMC has been delivered in Sokoto since 2015. As of week 37 when data collection started, 159 confirmed COVID-19 cases and 17 COVID-19 related deaths had been reported in Sokoto [13].

In Burkina Faso, the study was conducted in the urban health district of Bogodogo in the Centre region, and the rural health districts Léna and Dafra in the Hauts Bassins region. Bogodogo, an arrondissement of Ouagadougou, was added to the SMC campaign in 2019. SMC has been implemented in Dafra and Léna since 2017. The COVID-19 outbreak was concentrated around the two major cities Ouagadougou and Bobo Dioulasso. As of June $7^{\text {th }}, 414,28$ and 0 cases of COVID-19 were confirmed in Bogodogo, Dafra and Léna, respectively.

In Chad, the study was conducted in the urban health district of N'Djamena Sud in N'Djamena province, and the rural health districts of Massakory in Hadjer Lamis province and Guelendeng in Mayo Kebbi Est province. SMC has been implemented here since 2015. As of week 39 when data collection started, 1006 and 134 confirmed COVID-19 cases and 62 and 5 COVID-19 deaths had been reported in N'Djamena and Mayo-Kebbi Est provinces, respectively. There were no cases of COVID-19 reported in Hadjer Lamis province during the study period [14].

\section{Study sample}

The study was powered to estimate the proportion of community distributors in each country who adhered to infection prevention and control measures for SMC during the COVID-19 pandemic. Using the calculation for a cross-sectional study for proportions [15], a conservative estimate of $50 \%$ adherence, 
desired precision of $7.5 \%, a=0.05$, design effect of 1.4 [16] was used, with $10 \%$ increase applied to account for loss to follow-up or missing data. A minimum sample size of $n=263$ community distributors conducting SMC delivery was required for each country.

In Burkina Faso and Chad, health facilities were selected using stratified random sampling, to ensure a balanced number of urban and rural health facilities across the selected health districts. In Nigeria, multistage random sampling was used to select $a$ ) one senatorial district per state; $b$ ) three local government areas (LGAs) - two rural; one urban per state; c) health facilities.

All consenting community distributors aged 20 years or above participating in the SMC campaign at the selected health facility on the day of the observation were eligible to participate. Community distributors were selected randomly from a numbered list of all the community distributors working at the health facility.

For FGDs, community distributors were purposefully sampled from health facilities across the participating regions or LGAs in urban and rural areas to form male or female only groups. Each focus group discussion comprised of community distributors from one to two purposefully selected health facilities, depending on the number of community distributors available and where possible, male or female only groups were formed.

\section{Data collection}

We conducted observations of infection prevention control measures practiced by community distributors in the health facility and in the community. Our observational tool was adapted from published tools [17] [18], that were based on WHO guidelines $[19,20]$. Our tool captured each opportunity or "indication" where a specific infection prevention and control action should be followed by the community distributor in accordance with the job aid [9]. For some indications, equipment was also required. The observation tool was developed on a mobile data collection platform called SurveyCTO, translated to French and Arabic where required and piloted in all countries and geographical settings prior to data collection. Data collectors were trained to spend at least three hours observing the community distributor across three time points: (i) at the health facility at the start of the day when they received equipment for infection prevention and control, (ii) in the community (visiting at least five compounds) (iii) at the health facility at the end of the day.

We implemented strict standard operating procedures to ensure that data collectors were as discrete as possible and did not interfere with community distributors or families, to minimise the potential for bias created by the Hawthorne effect. To assure data quality, data collectors' skills were evaluated during a pilot prior to data collection, data submitted in SurveyCTO were checked daily, and for a sample (5\%) of observations in the community a supervisor observed the same community distributors at the same time as the data collector and inter-rater reliability was calculated. 
We conducted focus group discussions between 24 October and 20 November 2020 with community distributors who had participated in the campaign. The topic guide was designed to explore factors relating to quality and safety of SMC delivery: 1) training; 2) challenges adhering to safe delivery of SMC; 3) equipment availability; 4) acceptability of infection prevention and control measures; 5) knowledge and awareness of COVID-19. FGDs took place in the health facilities and were conducted by trained research assistants. To facilitate open discussion, where possible male and female CDs were split into separate discussion groups. To ensure data quality, all data collection procedures and tools were piloted for one day, with separate pilots for each local language. Discussions were audio recorded.

This study was carried out by employees of Malaria Consortium and the national malaria programme in each country, together with Oxford Policy Management (Nigeria), INSTECH (Burkina Faso) and Université de N'Djaména (Chad). Authors based in Abuja, Ouagadougou and N'Djamena were involved in monitoring and evaluation activities and those in the UK were responsible for supporting implementation research to improve programme performance and impact.

\section{Data analysis}

For descriptive statistics, frequencies and proportions were calculated for categorical data. Mean, standard deviation, and range were calculated for continuous data. Adherence to infection prevention and control was calculated at the indication level and then summed up to give a total adherence proportion by domain: 1) Hand hygiene; 2) Mask use; 3) Disinfection of SPAQ blister packs; 4) Waste management; 5) Safe distancing in the compound/household; 6) Ensure community distributors are healthy. For each indication, adherence was coded as "one" if the community distributors correctly performed the action or "zero" if they did not. If the community distributor did not have the necessary equipment to perform the action or the data collector could not see the action, it was coded as missing and excluded from the numerator and denominator. Definitions of indications, actions and the equipment required can be found in Additional File 1.

Equipment availability analysis was done at the level of the community distributor, expressed as a proportion and described at the LGA (Nigeria) or health district (Chad and Burkina Faso) level.

Quantitative data analysis was conducted in STATA/SE version 16.

Authors from each country team conducted a thematic analysis of data collected during FGDs [21]. Audio recordings were transcribed verbatim and translated into English. Transcripts were read by two members of each team to generate a coding list, which was then applied to all transcripts. Teams used qualitative data analysis software: NVivo (2018) and Dedoose version 8.0.35 (2018), or manual methods to code, manage and retrieve data. Country teams identified potential themes and collated all data relevant to each theme. Potential themes identified in each country dataset were then discussed together by the full 
team and consolidated into themes observed across all three countries. Theme names were agreed by the full team and each theme description was refined to elaborate the similarities and differences across the countries and substantiated with compelling participant quotes.

\section{Results}

In Nigeria, forty-one public health facilities were enrolled in Sokoto state, in urban (46.3\%) and rural (53.7\%) areas across Sokoto South (46.3\%), Tangaza (26.8\%) and Silame (26.8\%) LGAs. Of 259 community distributors observed, the majority (89.6\%) were females with a mean age of 28.7 years $(S D=10.2)$. The majority $(64.1 \%)$ had at least a secondary education and a mean of $3.2(S D=1.7)$ years' experience as a community distributor for SMC.

In Burkina Faso, forty-six public health facilities were enrolled in Bogodogo (47.8\%), Dafra (23.9\%) and Lena $(28.3 \%)$ health districts, in rural (56.5\%) and urban (43.5\%) areas. Of 252 community distributors observed, just over half $(51.6 \%)$ of CDs were male, with a mean age of 33.0 years $(S D=9.8)$. A quarter $(24.6 \%)$ of $C D s$ had at least a secondary education and a mean of $2.8(S D=1.7)$ years' experience as a community distributor.

In Chad, 35 public (31.4\%), private (11.4\%), community (28.6\%) and confessional (28.6\%) health facilities were enrolled in N'Djamena Sud (51.4\%), Massakory (31.4\%) and Guelendeng (17.1\%) health districts, in rural (48.6\%) and urban (51.4\%) areas. Of 266 community distributors observed, the majority $(73.7 \%)$ were male, with a mean age of 28.8 years $(S D=8.8)$. Under half $(42.1 \%)$ of community distributors had completed secondary education or above and they had an average of 3.1 years' $(S D=1.8)$ experience as a community distributor.

Inter-rater reliability between data collectors and their supervisors was high in Nigeria (kappa: 0.77, standard error: 0.02) and Burkina Faso (kappa: 0.76, standard error: 0.03) and moderate in Chad (kappa: 0.64 , standard error: 0.02 ).

In each country, eight focus group discussions (FGDs) were completed, across urban $(n=4)$ and rural $(n=4)$ areas with an equal number of male and female groups, with the exception of the rural areas in Burkina Faso, where four mixed male and female groups were formed.

\section{Observation of infection prevention and control adherence}

\section{Case study 1: Nigeria}

Equipment availability was variable between LGAs and for different types of equipment, with no distinct trends between rural and urban LGAs. Overall, $67.6 \%$ community distributors received hand sanitiser and $79.9 \%$ received at least one face mask, with a much lower proportion $(25.1 \%)$ receiving the recommended two new face masks. Availability of disinfecting wipes and bio-waste bags was low (Additional file 2). 
Adherence to mask use was high (506 [73.3\%] of 690 indications). Community distributors rarely washed their hands for the recommended 30 seconds (56 [3.5\%] of 1578 indications) but this increased when excluding length of time spent hand washing (578 [36.6\%] of 1578 indications). Hand sanitiser was used more frequently than soap and water. Community distributors rarely practiced exclusive safe distancing in the compound (211 [16.4\%] of 1279 indications) and community distributors' temperature was checked for 117 [22.6\%] of 518 indications. Due to low availability of disinfection wipes and biowaste bags, data on compliance with disinfection of SPAQ blister packs and waste management are inconclusive (Figure 2, Additional file 3).

\section{Case study 2: Burkina Faso}

Equipment availability varied by type of equipment and by health district. The majority of the community distributors received hand sanitiser across all three health districts ( $96 \%$ or above). For all other types of equipment, at least $10 \%$ more community distributors in the rural district of Lena were observed receiving equipment compared to the other two districts. Notably, $59.5 \%$ of community distributors received two or more new face masks, ranging from $44.1 \%$ in Dafra to $75.9 \%$ in Lena. Disinfecting wipes were available to at least a third of community distributors in all three health districts and bio-waste bags were available to over half of community distributors (Additional file 2).

Adherence to mask use was high (1168 [86.9\%] of 1344 indications). Adherence to hand washing for the recommended 30 seconds was low (165 [10.3\%] of 1606 indications) increasing substantially when excluding length of time spent hand washing (994 [61.4\%] of 1619 indications). Hand sanitiser was used more frequently than soap and water. Among those receiving wipes and biowaste bags, there was evidence of some adherence to disinfection of SPAQ blister packs (51 [17.4\%] of 294 indications) and waste management (102 [30.9\%] of 330 indications). Community distributors rarely practiced exclusive safe distancing in the compound (99 [7.9\%] of 1249 indications) and adherence to taking community distributors' temperature was very low [2.6\%] of 504 indications] (Figure 2, Additional file 3).

\section{Case study 3: Chad}

Equipment availability was variable between health districts and for different types of equipment, with no distinct trends between rural and urban districts. Overall, $89.8 \%$ community distributors received hand sanitiser and $92.9 \%$ received at least one face mask, with a much lower proportion (34.2\%) receiving the recommended two new face masks. Around a half of community distributors received disinfecting wipes (50.4\%) and bio-waste bags (45.9\%) (Additional file 2).

Adherence to mask use was high (1983 [81.4\%] of 2437 indications). Community distributors rarely washed their hands for the recommended 30 seconds (103 [3.4\%] of 3045 indications), increasing when excluding length of time spent hand washing (1362 [55.5\%] of 2453 indications). Hand sanitiser was used more frequently than soap and water. Among those receiving wipes and biowaste bags, there was 
evidence of some adherence to disinfection of SPAQ blister packs (68 [16.9\%] of 402 indications) and waste management (124 [41.1\%] of 302 indications). Community distributors rarely practiced exclusive safe distancing in the compound (135 [5.4\%] of 2512 indications) and adherence to taking temperature was quite low (79 [15.0\%] of 528 indications] (Figure 2, Additional file 3).

Table 1 Characteristics of community distributors enrolled in the study

\begin{tabular}{|c|c|c|c|c|c|c|}
\hline Characteristics & \multicolumn{2}{|c|}{$\begin{array}{l}\text { Nigeria } \\
(\mathrm{N}=259)\end{array}$} & \multicolumn{2}{|c|}{$\begin{array}{l}\text { Burkina } \\
\text { Faso } \\
(\mathrm{N}=252)\end{array}$} & \multicolumn{2}{|c|}{$\begin{array}{l}\text { Chad } \\
(N=266 *)\end{array}$} \\
\hline Age (years), mean [SD], min, max & \multicolumn{2}{|c|}{$\begin{array}{l}28.7(10.2) \\
18,70\end{array}$} & \multicolumn{2}{|c|}{$\begin{array}{l}33.0(9.8) \\
20,63\end{array}$} & \multicolumn{2}{|c|}{$\begin{array}{l}28.8(8.8) \\
20,70\end{array}$} \\
\hline \multicolumn{7}{|l|}{ Age (years), n (\%) } \\
\hline$<30$ & 168 & 64.9 & 101 & 40.1 & 162.0 & 60.9 \\
\hline $30-49$ & 73 & 28.2 & 134 & 53.2 & 92.0 & 34.6 \\
\hline$\geq 50$ & 18 & 6.9 & 17 & 6.8 & 11.0 & 4.1 \\
\hline \multicolumn{7}{|l|}{ Sex, n (\%) } \\
\hline Female & 232 & 89.6 & 122 & 48.4 & 69 & 25.9 \\
\hline Male & 27 & 10.4 & 130 & 51.6 & 196 & 73.7 \\
\hline \multicolumn{7}{|l|}{ Education, n (\%) } \\
\hline No education & 21 & 8.1 & 0 & 0.0 & 13.0 & 4.9 \\
\hline Arabic/Islamic school & 31 & 12.0 & 1 & 0.4 & 17.0 & 6.4 \\
\hline \multirow[t]{2}{*}{ Primary } & 5 & 1.9 & 27 & 10.7 & 17.0 & 6.4 \\
\hline & 11 & 4.2 & 37 & 14.7 & 11.0 & 4.1 \\
\hline Some secondary & 25 & 9.7 & 125 & 49.6 & 95.0 & 35.7 \\
\hline Completed secondary & 87 & 33.6 & 21 & 8.3 & 39.0 & 14.7 \\
\hline Some tertiary & 26 & 10.0 & 34 & 13.5 & 54.0 & 20.3 \\
\hline Completed tertiary & 53 & 20.5 & 7 & 2.8 & 19.0 & 7.1 \\
\hline $\begin{array}{l}\text { Years of experience as a community distributor, } \\
\text { mean (SD) min, max }\end{array}$ & \multicolumn{2}{|c|}{$3.2(1.7), 1,7$} & \multicolumn{2}{|c|}{$2.8(1.7), 0$} & \multicolumn{2}{|c|}{$3.1(1.8) 0,7$} \\
\hline
\end{tabular}




\section{Findings from focus group discussions with community distributors}

\section{Acceptability of COVID-19 infection prevention and control measures}

Community distributors in urban and rural areas of Burkina Faso, Chad and Nigeria viewed the infection prevention and control measures favourably, expressing that the equipment gave them confidence and motivation to participate in the SMC campaign despite the pandemic, typically:

"Truly this prevention that they brought is proper and it has given us peace of mind, we know what to do because before, everyone was afraid of this sudden situation. This precaution has given us peace of mind, on top of that they added sanitizer and facemask, so we feel confident working with them... even if we get infected, we will not spread it or bring it home or to our environment" (Nigeria_Sokoto_ Urban Female_02).

"In any case...I come back to what she said. It's a good thing as it allows us to protect ourselves, we protect ourselves against Covid-19 and other diseases as well. And it makes us clean." (Burkina Faso_FGD2_F_Trame D'Accueil)

"Which reassures us, during this ...at the time of the distribution, we had all the materials such as hydroalcoholic gel, gloves, and then the mask, we had all that, that's what reassures us..." (Chad_FDG2_M_Kamerom)

However, many community distributors were still fearful. In Burkina Faso, although community distributors generally agreed with infection prevention and control measures, many still expressed 'a lot of fear' of being infected with COVID-19. In Nigeria, male distributors admitted being initially fearful of becoming infected but when they were trained and assured that infection prevention and control equipment would be provided, they were put at ease. In Chad, some distributors felt compelled to use the mask for fear of being reprimanded by the police. For additional quotes please see Additional File 4.

\section{Feasibility of implementing infection prevention and control measures}

\section{Impact of infection prevention and control measures on community distributor's workload}

In Burkina Faso and Nigeria, community distributors acknowledged that the biggest impact on their workload was not implementing the infection prevention and control measures, rather the time taken to explain the changed circumstances of the SMC campaign, convince reluctant caregivers about the need for additional measures and address caregiver concerns about the campaign during COVID-19. In Burkina 
Faso and Nigeria, distributors found communicating the need for infection prevention and control measures to illiterate caregivers particularly challenging. In Burkina Faso, distributors indicated that some caregivers did not fully understand the practices, especially the need for hand washing; some caregivers thought community distributors asked them to wash their hands because they were dirty. In Chad, distributors mentioned that delivering SMC during the pandemic had led to some mistrust; some caregivers were afraid that distributors would bring COVID-19 into the household and refused compound entry on the pretext that everyone had been asked to stay at home.

"Most of them are aware that it is for their own protection. But for example, when you arrive in a compound where everybody is illiterate, it's a complicated matter... We explain them, they understand, but can't really comply with the rules." (Burkina Faso_FGD2_F_ Trame D'Accueil)

"You arrive at someone's house and you have to wash your hands before giving medications; this can frustrate the person because it implies that their hands are not clean." (Burkina Faso_FGD5_F_Secteur 24)

"The work you can finish in 30minutes for example when you come and do your introduction, it will add more minutes instead of maybe 30 minutes, it will increase to 50 minutes this is because you will have to go through the measures and tell them about it step by step" (Nigeria_Sokoto, Urban_female_02)

"Corona affected the distribution of CPS [SMC] a bit in that it caused a bit of mistrust [...] parents are a little afraid that we are bringing this virus to them to distribute so they are afraid when we are approach to them for the distribution of drugs and others even outright refuse this contact. Even if we respect the distancing to give but they are afraid that corona is there so when we knock on the door many times, they respond violently, stuff like that, that's my opinion" (Chad_FGD8_M_Moursal)

As a result, distributors described working additional hours in order to reach their targets for drug administration, which they said was exhausting. Most mentioned the time taken for hand hygiene for themselves and the caregiver before administering the first dose, and time to put on face masks; but they did not seem concerned by this and mostly they regarded the tasks as necessary and feasible to do. In contrast, community distributors in Chad felt that having to adhere to the infection prevention and control measures had a negative impact on their work and encroached on work time to the extent they felt forced 'to do a double job'; administering SMC and raising awareness about COVID-19.

"How coronavirus affected work, this is called, overwork. Because you have to start raising awareness first. It is already taking time. Then you have to take the hygiene measures before doing the actual work as it was told to do. This makes it an overload of work for us." (Burkina Faso_FGD1_Mixed_Peele)

"You will see a particular work that you are supposed to round up in 5minutes you will end up rounding up in 10minutes, seriously houses that you are expected to cover per day, because of all those issues you will be unable to complete it" (Nigeria_Sokoto_Rural_Female)

"... the work is heavier, that's what I was saying, it's that workload, because before, we were supposed to simply administer only the drugs, but here now it's as if we have a double mission like that, it is necessary 
and, to do the CPS [SMC] and it is also necessary to make the awareness of covid so that it weighs down a little and it plays on time. Yes, it is a challenge" (Chad_FGD7_F_Moursal)

Distributors in rural Sokoto, Nigeria explained that the state media and local town criers played a vital role in imparting information about COVID-19 prevention, creating awareness before the SMC campaign and allaying caregivers' fears. Despite this, community distributors reported that some caregivers refused to greet them, or requested they wait outside the compound as they did not understand the rationale for the infection prevention and control measures or were worried that the visit to their home posed a threat to their safety.

\section{Community distributors found face masks uncomfortable to wear}

In all three countries, community distributors reported that wearing face masks throughout the working day was challenging. In Burkina Faso and Nigeria, many pointed out they had already been wearing masks for health reasons or to prevent inhalation of dust in arid regions; however, having to wear masks continuously throughout the day was their main concern. Typical discomforts community distributors experienced included:

"There are several difficulties such as breathing problems associated with wearing masks. It happens that often you do not manage to get oxygenated air properly..." (Burkina Faso_FGD6_Mixed_Yegueresso)

"yes, honestly we somehow faced challenge because...using the face mask for the fact that we were not used to it before, even though we were using it in the past but now we use it often, we put it on during work, even after until we go back to the house before they say we can remove it, we need to have it on like 6 to 7 hours, we are not used to this duration" (Sokoto, Urban_female_04).

"Because coronavirus is there, that's how we wear the mask to distribute SMC. It squeezes and it hurts our ears. We can't breathe, even it was coronavirus that brought it all. Wearing the mask there is annoying" (Chad_FDG1_F_Kamerom)

Some distributors mentioned that supervisors conducting spot checks facilitated consistent and continuous mask-wearing throughout the day.

"Some, we leave from the house with. So it's already (whispers) from the house as soon as you leave at 6am sometime before you get there. It wasn't easy, why, because we're not used to it a few times it's like it suffocates us, it's a reflex by the way. We used to want to shoot like that first ... and when we see the supervisors behind us first we try to pull that out. It wasn't easy because the time is long and to stay like that with there, suddenly it suffocates us so it was not easy for me" (Chad_FDG5_F_Abena)

"Some people demanded that it be taken off because they didn't understand what we were saying. In order for them to understand us and for the work to go well we had to take off the mask. So we had to 
take it off while being careful not to be seen by a supervisor. So it complicated the work a lot because we were doing it in secret, so we had to do it quickly". (Burkina Faso_FGD5_F_Secteur 24)

Barriers to consistent mask-wearing included complaints from community members about not being able to hear them clearly when speaking, being unrecognisable from a distance, and children being afraid of them. In all countries, distributors reported deliberately lowering or removing their mask when giving instructions for the drug administration but keeping the mask on to administer SMC drugs. Further to this, in Nigeria, distributors mentioned that caregivers demanded to see their face when administering SMC to their children; masks were thought to be a deliberate disguise to protect distributors if adverse events occurred.

"Well, we had challenges especially entering the houses, some parents once you knock on their door and greet them, they will start saying "you just come to give our children drugs without us knowing who you are?" So you see we will have to remove our facemask for recognition, they will even ask to know if we are the people that came the last month and we reply them, you see it is also a challenge" (Nigeria_Sokoto, Urban_female_04).

In Chad and Nigeria, community distributors were harassed when wearing masks - some recounted children running after them chanting, 'the corona people', which attracted a lot of attention and made them feel uncomfortable. In Chad, children reportedly shouted at community distributors wearing masks, as they were not familiar with face coverings.

\section{Cultural norms made it difficult to adhere to safe distancing}

In all three countries, cultural norms and traditions mean people are accustomed to greeting each other with physical contact and often spend time together in close proximity. As a result, community distributors described distancing as the most difficult measure to adhere to:

"We were uncomfortable because we said not to greet by shaking hands with people we are already used to, as we chat and laugh together, without realizing. But if we are prevented from doing this, will we be able to be comfortable? Obviously, we won't be comfortable. ((Laughter)) This is the aspect that is complicated". (Burkina Faso_FGD6_Mixed_Yegueresso)

"Like that of social distancing, since you know the person and they know us, it is hard not to come, sit and discuss and even shake [hands]" (Nigeria_Sokoto_Rural_male)

"It is not easy as she says there true because as a good Chadian there you have to shake hands, because when you only greet like that, the person tells you... so you have to shake hands and given the illness, in doing this some people find that you may be neglecting them..." (Chad_FDG5_F_Abena)

In Nigeria, community distributors mentioned that it was difficult to observe safe distancing because they sometimes forgot, were influenced by caregivers or community members' perceptions, and in a few 
instances space constraints in households precluded it. In rural Sokoto state, caregivers seemed to interpret safe distancing as community distributors' irritation or anxiety about contracting COVID-19 from them.

Some community distributors found ways to work around the safe distancing measures. For example, in Nigeria, female distributors explained how a 1-metre distance was more comfortable and feasible, so long as a face mask is worn and proper hand hygiene observed. In Burkina Faso, distributors occasionally felt obliged to shake the hands of the elderly to appear respectful, although they emphasised that they sanitised their hands immediately after. In addition, distributors in Burkina Faso were tasked with measuring nutritional status of children alongside administering SMC; using upper arm circumference in under-5s necessitated touching the child's arm, but distributors were keen to emphasize that they disinfected the Shakir (measurement) strip before and after each use.

\section{Hand hygiene adherence was sub-optimal}

Community distributors recognised the importance of hand hygiene and considered hands an easy source of COVID-19 infection and transmission from touching the mouth and nose with contaminated hands. However, distributors in all three countries admitted that hand hygiene was not done as frequently or for as long as stipulated in guidelines. In Burkina Faso and Chad, distributors talked about forming a habit, although many admitted that early in the campaign they often forgot. In rural Sokoto state, Nigeria, distributors mentioned that unannounced visits by supervisors motivated them to adhere to the guidelines; some also regarded hand hygiene as a mandatory instruction and so kept to this.

"At the beginning, it was not easy at first. But in everything, the more you do it, the more you get used to it. So it was like that." (Burkina Faso_FGD1_Mixed_Peele)

"Well, it's a matter of habit eh, these are not the measures that we are used to doing but given the arrival of this disease we knew that it is really annoying, it is really worrisome then is to enable us to protect against disease. But most, most of the population does not want to apply these measures at all" (Chad_FDG6_M_Abena)

"Yes ma since it's a promise you have taken upon yourself, you have to follow it diligently because they were appropriate and if you don't want any problem, you just have to adhere to the directives" (Nigeria_Sokoto_Rural_female_05)

In Nigeria, distributors explained that although they were happy to practice hand hygiene, they found it difficult to adhere to 30 seconds each time. A few also reported that alcohol-based sanitisers caused unpleasant skin irritations and frequent application made their hands dry. A minority expressed concern that alcohol-based sanitiser went against religious rulings that forbid use of alcohol, and they were more likely to use soap and water. 
"I can only say I did my best with the hand hygiene, but I am not certain about adhering to the 30 seconds rule" (Nigeria_Sokoto_South_female_02).

"Some Community distributor do not use the hand sanitizer. They do say it contains alcohol and so on and that because of that, their prayer is affected" (Nigeria_Sokoto, Urban_male_01)

Availability of soap and water in households was an important challenge in all three countries.

Distributors in Burkina Faso recounted having to share soap and sanitiser with households as caregivers often did not have any, and in Chad distributors found it easier to use hand gel in communities as it 'is difficult to find soap in some homes'.

\section{SMC administration by caregivers during the coronavirus pandemic}

In all three countries community distributors reported having to assist caregivers to administer the first doses of SP and AQ. In Burkina Faso, some caregivers recognized that their children would not accept the medicine if they had to administer it and preferred the distributor do it.

"There it is not easy, that is to say, she does not follow exactly what she is asked to do. We might ask to bring, how shall I put it, take the medicine and, take two pills out for the first dose, sometimes she doesn't do exactly what she's asked to do -there, maybe she even removes three. Effectively we keep saying that no, no it should have been two and not three..." (Chad_FGD8_M_Moursal)

"As they know that it's to help them that we respect the barrier measures, so some children would refuse to drink the medication if their own parents had to give them the medication. So this new method that has been adopted is a bit complicated! Unless we tease them to say we're going to give them a shot or something else, they will not take the medication with their parents!" (Burkina Faso_FGD2_F_Trame D'Accueil)

In Burkina Faso and Nigeria, distributors also mentioned coaxing children to take the drug by singing to them or teasing them. Although some children were happy to receive the drugs from caregivers, community distributors had to step in when children cried or ran away from parents; distributors felt that this tendency was more prevalent among older children who recalled polio immunisation campaigns. In both countries, community distributors carried sweets to persuade children to swallow the drugs, although this resulted in children refusing the drugs from their caregivers.

\section{Discussion}

This study assessed adherence to and perceptions of infection prevention and control measures for delivery of SMC during the COVID-19 pandemic. Adherence varied across different domains of infection prevention and control, and discussions with community distributors provide insights into acceptability of the measures, barriers and facilitators to their use as well as challenges to SMC administration by caregivers. 
Observations found that adherence to hand hygiene was sub-optimal in all countries. As a primary measure to reduce the spread of COVID-19 [22] and a pre-existing recommendation for implementation of SMC, this is a concerning albeit unsurprising finding. Studies assessing health facility worker compliance with hand hygiene in Tanzania [17] and Kenya [18], found similarly low levels of compliance: $6.9 \%$ and $2.3 \%$ respectively, although this was not in a pandemic context. FGDs highlighted that community distributors found it time consuming, they sometimes forgot and it was uncomfortable to wash hands for the recommended time period, particularly when using hand sanitiser. It is worth noting that the guidelines deliberately recommended 30 seconds to increase the likelihood of community distributors washing for at least 20 seconds [Personal communication, SMC Programme Director], which was the standard international guidance at the time [23]. Future campaigns could increase spot checks by supervisors and emphasise the importance of washing hands for at least 20 seconds during training. To address shortages of running water and soap at compounds, future campaigns could try to improve availability of hand sanitiser and soap for community distributors.

The majority of community distributors in all three countries were observed wearing their mask. Qualitative data suggest community distributors found mask wearing uncomfortable but the presence of supervisors conducting spot checks increased compliance. However, significant barriers to adherence were raised in FGDs; masks made it difficult to verbally communicate SMC administration instructions clearly, many children were fearful of the masks and community distributors were harassed. There is little published evidence relating to mask-wearing in community health campaigns, but future SMC campaigns could consider mask "rest" periods. For example, when travelling outdoors between compounds and other times when community distributors are able to maintain a two-metre distance. However, it would be critical to emphasise importance of hand washing after touching a used mask, should this be implemented.

Data from observations suggest that practicing safe distancing exclusively in the compound was challenging in all three countries. A recent paper discusses how social values including solidarity between extended family groups makes respecting social distancing during COVID-19 in west African societies particularly challenging [24]. Community distributors for SMC are often members of the community and are known to the families, making it especially difficult to respect both social values and safe distancing. Furthermore, in space-constrained areas, qualitative data suggested it was impossible to maintain this distance and some community distributors would try to maintain a shorter distance of one meter instead. Future campaigns could consider "graded recommendations" [25] for distancing for certain scenarios such as whether the community distributor is indoors or outdoors, and whether they are wearing a mask.

In the context of the pandemic, normal community engagement activities such as sensitization meetings with local leaders and community members and dissemination of information through town announcers and media were cancelled or adapted [26]. Qualitative data suggest that there was a lack of awareness in the community about the importance of infection prevention and control measures and this greatly impacted on the workload of community distributors, particularly when explaining the measures to illiterate caregivers. Future community engagement approaches should start as early as possible and 
consider visual and audible information such as posters and radio jingles to support illiterate caregivers to understand the measures. This has the potential to reduce the additional workload experienced by community distributors during the campaign which is essential for their wellbeing, motivation and retention.

Due to the lack of in-person training, participatory adult training techniques, for example, in-person demonstrations, role plays and trainer observation of trainees completing tasks were not used widely in 2020 [26]. These are recognised approaches for training community health volunteers [27], and where possible, should be prioritised for future campaigns to increase knowledge of and cooperation with the infection prevention and control measures and develop community distributors' ability to communicate the measures with caregivers. It is promising that community distributors felt more confident with the protective measures; this is something that could be reinforced by identifying role models to help other community distributors feel less fearful.

The international guidance and standards for safe implementation of SMC rapidly developed for the 2020 campaign were based on the best available evidence at the time, including the relative contribution of different transmission pathways to the spread of COVID-19. It is evident that there were differences between these and national infection prevention and control standards. For example, in Burkina Faso, community distributors were also responsible for anthropometric measurements to assess nutritional status which compromised efforts to ensure safe distancing. Future campaigns should work with relevant stakeholders such as the national malaria programme to ensure adaptation and implementation of international guidelines, where possible.

The observational tool used in this study was based on direct observations which could have led to community distributors altering their behaviour because they are being observed (Hawthorne effect). The observation tool was also restricted to the SMC infection prevention and control activities stipulated in the job aid and based on international guidance. Due to the rapidly changing nature of the COVID-19 pandemic, there were local adaptations to the types of equipment used and the order with which some of the SMC activities took place that could not be captured in the data collection tool. Efforts were made to accommodate local adaptations where possible in the pilots, but it is plausible that the indicators reported here underestimate the actual compliance where equivalent equipment were used or infection prevention and control events took place at a different time to when they were observed. Mask use should have been practiced continuously but was measured at discrete time points and could be an overestimation of the actual compliance over time. Due to low availability of biowaste bags and disinfecting wipes in Sokoto State, Nigeria, there was a high proportion of missing data for these domains, making the adherence results inconclusive. Finally, the tool did not capture availability of the infrared thermometer at the health facility or soap and water at the health facility or compound, and results should be interpreted with this caveat in mind.

It is worth noting that the quantitative findings presented here relate to infection prevention and control adherence and equipment availability in the latter two cycles of SMC. Readers should interpret the 
quantitative findings within these boundaries. Qualitative findings allow the reader to gain insight into additional issues occurring throughout the four-month campaign.

\section{Conclusion}

Our findings suggest that the infection prevention and control measures were acceptable to community distributors. However, key barriers to adherence were identified and equipment availability was inconsistent. Our findings demonstrate that no single infection prevention and control measure can be adhered to perfectly and therefore a combination of measures is needed. Future campaigns should consider how to make the infection prevention and control measures more feasible to implement. Early awareness raising and further training on how to communicate the importance of the infection prevention and control measures would help relieve the burden placed on community distributors to sensitise the communities. Harmonising international and national guidelines before and during the campaign is important.

\section{Declarations}

\section{Ethical approval and consent to participate}

Ethical approval for the study was provided Ministry of Health, Sokoto State on 29 July 2020 (ref. SMH/1580/V.IV); le Comité d'éthique pour la recherché en santé (CERS, Burkina Faso) on 12 August 2020 (ref. 2020-8-143) and le Comité National de Bioéthique du Tchad (CNBT) on 21 September 2020 (ref. 0190).

Written informed consent was provided by all participants of this study.

\section{Consent for publication}

Not applicable.

\section{Availability of data and materials}

The data that support the findings of this study are available from the corresponding author upon reasonable request.

\section{Competing interests}

The authors declare that they have no competing interests. 


\section{Funding}

This research is funded through philanthropic donations, mainly received as a result of being awarded Top Charity status by GiveWell, a non-profit organisation dedicated to finding outstanding giving opportunities through in-depth analysis.

\section{Authors' contributions}

CW, AP, OO, AM, AT, CS, JBO, LD, MD, HS and KB conceived of and designed the study. AP, GO, EA, AM, HB, VA, CS, AT, IZ, YDC led acquisition of data. CW, GO, AM, VA, AT, LD, MD and HS analysed data. All authors interpreted the findings. CW and HS drafted the manuscript and all authors reviewed subsequent versions and approved the final version for submission.

\section{Acknowledgments}

The authors would like to thank all study participants who took part in the study. We also would like to express our gratitude to Babatunde Akano, Festus Okoh, Benoit Sawadogo, Dr Kodbessé Boulotigam, Dr Rakiswendé Serge Yerbanga and Moussa Zongo for their generous support with implementing the study and supporting data analysis. Finally, we extend thanks to Simon Peter Kigozi for his support with additional data analysis.

\section{References}

1. Wang, J., et al., Preparedness is essential for malaria-endemic regions during the COVID-19 pandemic. The Lancet, 2020. 395(10230): p. 1094-1096.

2. World Health Organization, COVID-19: Operational guidance for maintaining essential health services during an outbreak. 2020.

3. World Health Organization. Tailoring malaria interventions in the COVID-19 response. 20203 April 2020; Available from: https://www.who.int/publications/m/item/tailoring-malaria-interventions-inthe-covid-19-response.

4. World Health Organization, WHO policy recommendation: seasonal malaria chemoprevention $(S M C)$ for Plasmodium falciparum malaria control in highly seasonal transmission areas of the Sahel sub-region in Africa. 2012: Geneva, Switzerland.

5. Meremikwu, M.M., et al., Intermittent preventive treatment for malaria in children living in areas with seasonal transmission. Cochrane Database of Systematic Reviews, 2012(2). 
6. ACCESS-SMC Partnership, Effectiveness of seasonal malaria chemoprevention at scale in west and central Africa: an observational study. Lancet, 2020. 396(10265): p. 1829-1840.

7. Malaria Consortium, Malaria Consortium's seasonal malaria chemoprevention program: Philanthropy report 2020. 2021.

8. Roll Back Malaria Partnership to End Malaria, Adapting seasonal malaria chemoprevention in the context of COVID-19: operational guidance. . 2020.

9. Malaria Consortium. Steps for using Infection Prevention and Control to Deliver SMC during COVID-19 Pandemic. 2020; Available from: https://www.malariaconsortium.org/gallery-file/06170924-10smc_covid19jobaid.pdf.

10. Kuehne, A., et al., Impact and Lessons Learned from Mass Drug Administrations of Malaria Chemoprevention during the Ebola Outbreak in Monrovia, Liberia, 2014. PLoS One, 2016. 11(8): p. e0161311.

11. Pringle, J., et al. Mass drug administration of antimalarials in the Ebola epidemic in Sierra Leone and Liberia. MSF Scientific Day; Royal Society of Medicine, London; May 7-8, 2015. in MSF Scientific Day. 2015. Royal Society of Medicine, London.

12. Donabedian, A., The Quality of Care: How Can It Be Assessed? JAMA, 1988. 260(12): p. 17431748.

13. Nigeria Centre for Disease Control. COVID-19 SITUATION REPORT 192, Monday 7th September 2020. 2020 18/02/2021]; Available from: https://ncdc.gov.ng/diseases/sitreps/? cat=14\&name=An\%20update\%20of\%20COVID-19\%20outbreak\%20in\%20Nigeria.

14. République du Tchad: Ministère de la sante publique et de la solidarité nationale. COMMUNIQUE: la situation épidémiologique du covid-19 au Tchad. 2020 08/04/2021]; Available from: http://santetchad.org/.

15. Pourhoseingholi, M.A., M. Vahedi, and M. Rahimzadeh, Sample size calculation in medical studies. Gastroenterology and hepatology from bed to bench, 2013. 6(1): p. 14-17.

16. Rowe, A.K., et al., Design effects and intraclass correlation coefficients from a health facility cluster survey in Benin. International Journal for Quality in Health Care, 2002. 14(6): p. 521-523.

17. Powell-Jackson, T., et al., Infection prevention and control compliance in Tanzanian outpatient facilities: a cross-sectional study with implications for the control of COVID-19. Lancet Glob Health, 2020. 8(6): p. e780-e789.

18. Bedoya, G., et al., Observations of infection prevention and control practices in primary health care, Kenya. Bulletin of the World Health Organization, 2017. 95(7): p. 503-516. 
19. World Health Organization, Revised injection safety assessment tool: tool C - revised. 2008, World Health Organization: Geneva.

20. World Health Organization. Hand hygiene-Observation Tool. 2009 [cited 2020; Available from: https://www.who.int/teams/integrated-health-services/infection-prevention-control/hand-hygiene/toolsand-resources.

21. Braun, V. and V. Clarke, Using thematic analysis in psychology. Qualitative Research in Psychology, 2006. 3: p. 77-101.

22. World Health Organization, Recommendations to Member States to improve hand hygiene practices to help prevent the transmission of the COVID-19 virus. 2020: Geneva, Switzerland.

23. World Health Organization, Water, sanitation, hygiene, and waste management for SARS-CoV-2, the virus that causes COVID-19. 2020.

24. Sougou, N.M., et al., What community-based public health approaches in West Africa for COVID19 epidemic? A reflection based on the African socio-cultural context. Pan Afr Med J, 2020. 35(Suppl 2): p. 91.

25. Jones, N.R., et al., Two metres or one: what is the evidence for physical distancing in covid-19? BMJ, 2020. 370: p. m3223.

26. Malaria Consortium, Implementing mass campaigns during a pandemic: What we learnt from supporting seasonal malaria chemoprevention during COVID-19. 2020.

27. Vizeshfar, F., M. Zare, and Z. Keshtkaran, Role-play versus lecture methods in community health volunteers. Nurse Education Today, 2019. 79: p. 175-179.

\section{Figures}



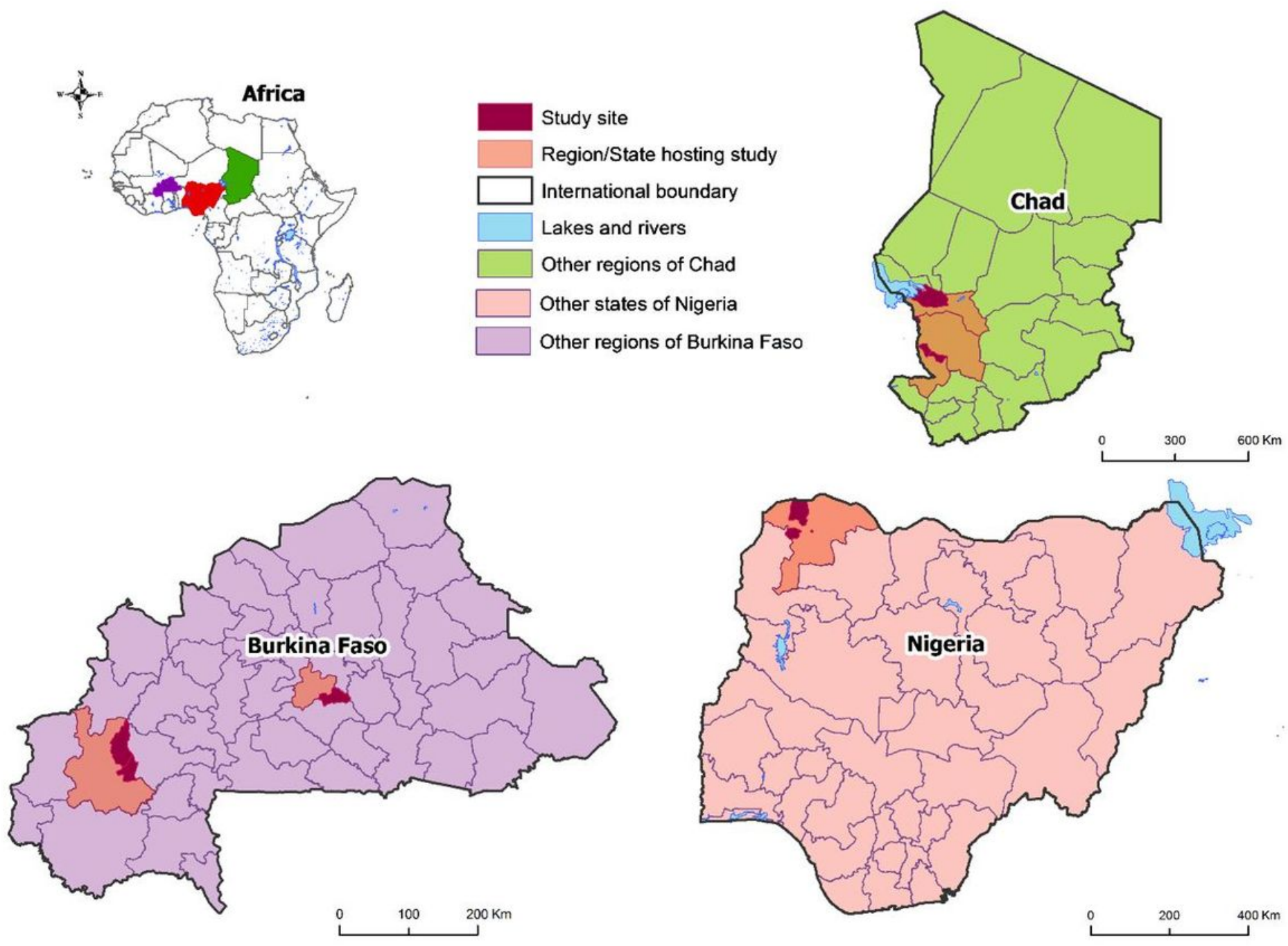

Figure 1

Map of the study sites and regions/states hosting the study 


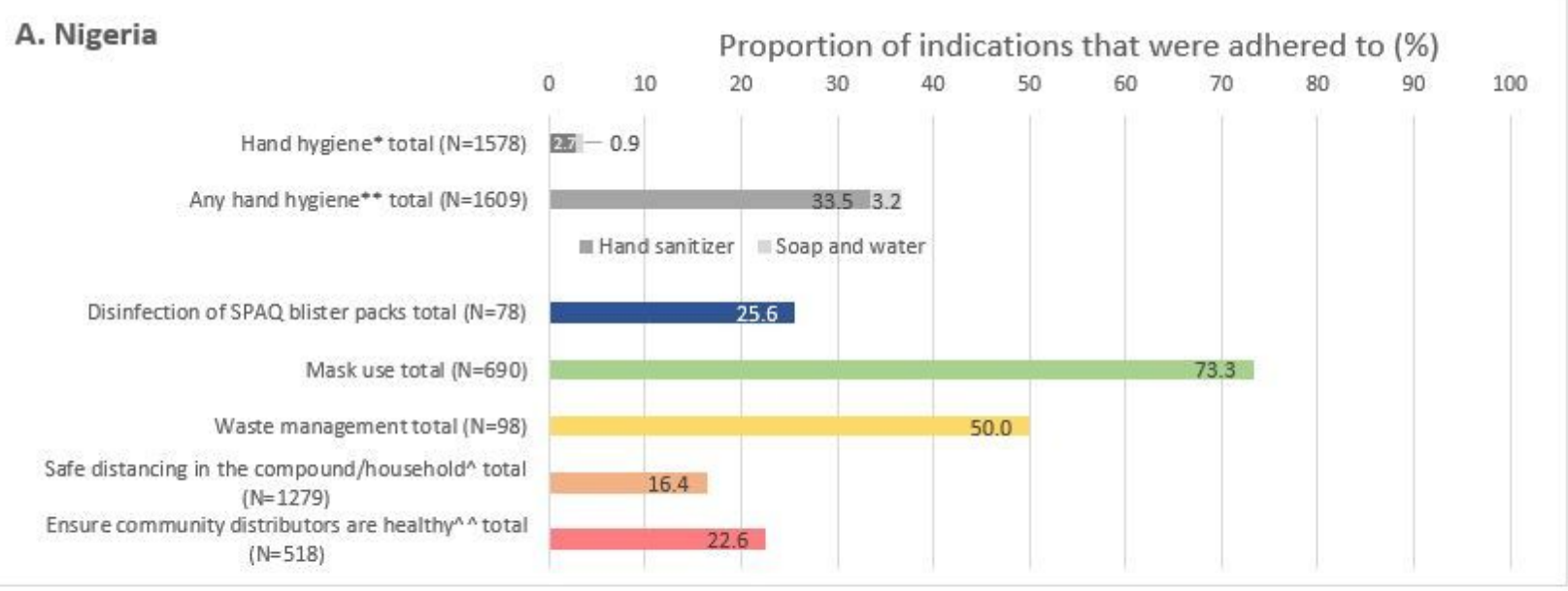

\section{B. Burkina Faso}

Proportion of indications that were adhered to (\%)

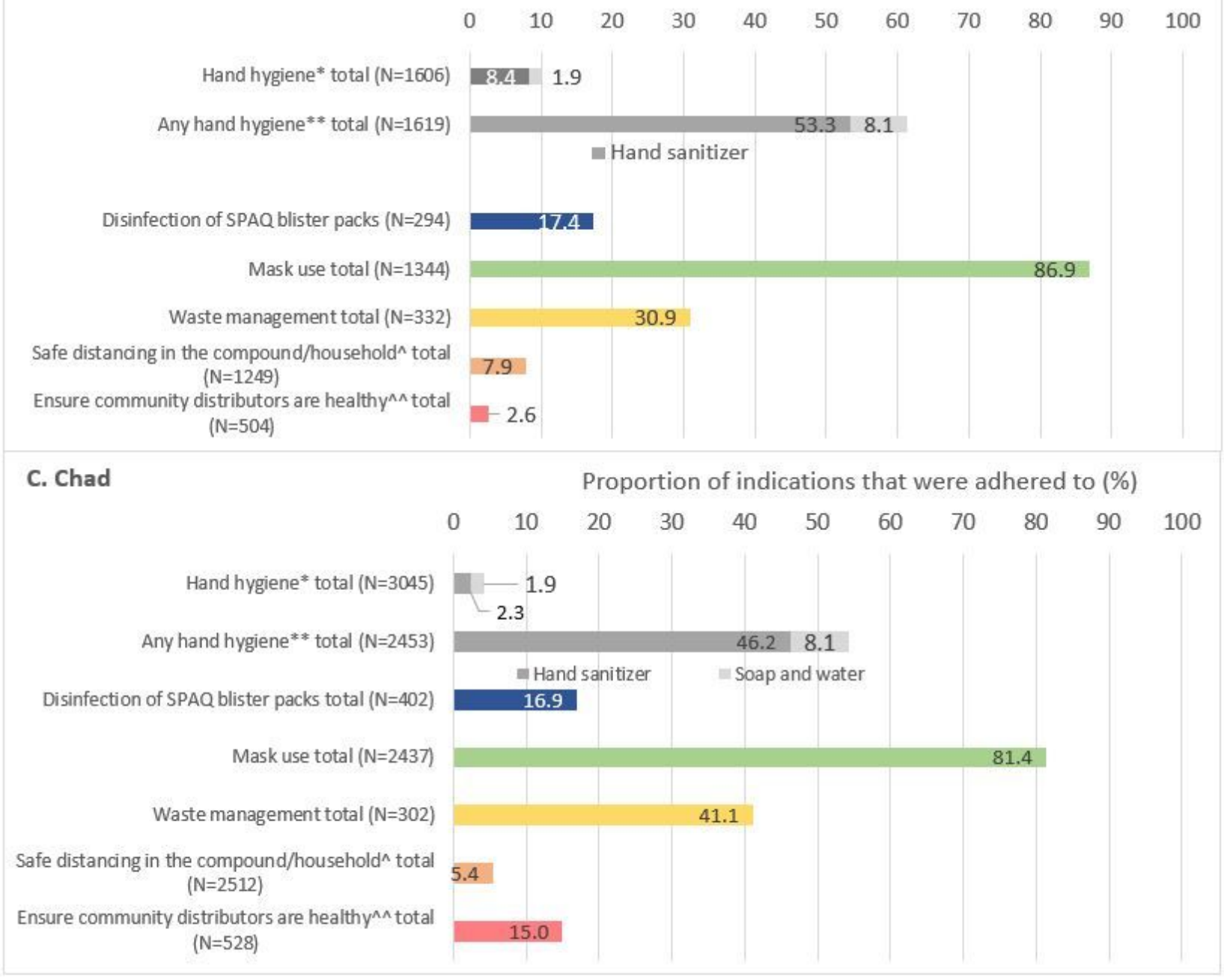

\section{Figure 2}

Adherence to infection prevention and control practices, by domain *Washed hands with soap and running water or hand sanitiser for $\geq 30$ seconds; $* \star W a s h e d$ hands with soap and running water or hand sanitiser; ^ during triage AND when determining age eligibility AND SPAQ eligibility AND SPAQ administration AND instructions AND messages; ${ }^{\wedge \wedge}$ Take temperature with infrared thermometer at start and end of the day at the health facility 